\title{
Fixed-point-free 2-finite automorphism groups
}

\author{
Theo Grundhöfer And Enrico Jabara
}

\begin{abstract}
A fixed-point-free group $G$ of automorphisms of an abelian group is shown to be locally finite if any two elements of $G$ generate a finite subgroup.
\end{abstract}

Mathematics Subject Classification (2010). Primary 20F28;

Secondary 20B22, 20F50, 12K05.

Keywords. Fixed-point-free automorphism, Sharply 2-transitive group, Nearfield.

A group $G$ of automorphisms of a group $(A,+)$ is called fixed-point-free, if $g(a) \neq a$ for $1 \neq g \in G$ and $0 \neq a \in A$. One says that a group $G$ is $n$-finite, if any $n$ elements of $G$ generate a finite subgroup; local finiteness means that this holds for every positive integer $n$. We prove the following results.

Theorem 1. Let $G$ be a fixed-point-free group of automorphisms of some abelian group. If $G$ is 2-finite, then $G$ is locally finite. Moreover, $G$ is countable. In fact $G / G^{\prime}$ and $G^{\prime} / G^{\prime \prime}$ have locally cyclic subgroups of index at most 2 , and $G^{\prime \prime}$ is finite and isomorphic to $\mathrm{SL}(2,5)$ or to the quaternion group $Q_{8}$ of order 8 or $\left|G^{\prime \prime}\right| \leq 2$.

With the modified assumption that $G$ is 1-finite, i.e., periodic (or even of finite exponent), $G$ need not be locally finite, as the examples at the end of this paper show. For groups $G$ as in Theorem 1, the subgroups generated by all elements of prime order are characterized in Sozutov [9, Theorem 1]. Our theorem does not readily follow from Theorem 3 in [9], since that Theorem 3 imposes a weak version of 2-finiteness on the whole Frobenius group, and not just on the Frobenius complement.

Corollary 2. Let $N$ be a nearfield such that the multiplicative group $N^{\times}$of $N$ is 2-finite. Then $N$ is a locally finite nearfield. 
Corollary 3. Let $G$ be a sharply 2-transitive permutation group, and assume that $G$ is 2 -finite. Then $G$ is locally finite, and if $G$ is infinite, then

$$
G \leq \operatorname{A\Gamma L}(1, F):=\{x \mapsto a \cdot \alpha(x)+b \mid a, b \in F, a \neq 0, \alpha \in \operatorname{Aut} F\}
$$

for some locally finite field $F$.

The locally finite nearfields are known in some detail (see [3] or [11, IV]). Corollaries 2 and 3 extend the main results of [10]. According to Zassenhaus [13, Satz 17], there are exactly seven finite sharply 2-transitive groups that are not subgroups of $\mathrm{A \Gamma L}(1, F)$ for a finite field $F$; see also $[7,20.3]$.

By a result of Jacobson, every skew field with periodic multiplicative group is locally finite (and commutative); see [1, Theorem 3.9.5]. Thus one might conjecture that the two corollaries hold with the weaker assumption of periodicity instead of 2-finiteness.

Proof of Theorem 1. The group $G$ contains at most one involution $g$, since $g$ fixes $a+g(a)$ for every element $a$ of the abelian group acted on, hence $a+g(a)=0$, and $g$ is the inversion and belongs to the center of $G$. This implies that the finite 2-subgroups of $G$ are cyclic or generalized quaternion groups; see [8, 5.3.6] or $[12,5.3 .2]$. For odd primes $p$, all finite $p$-subgroups of $G$ are cyclic (see $[8,10.5 .5]$ and [4, Lemma 2.6], or [14, Lemma 2]).

Every finite subgroup $H$ of $G$ has a normal subgroup $Z$ such that all Sylow subgroups of $Z$ are cyclic and

(a) $H^{\prime \prime}$ is trivial and $|H: Z| \leq 2$, or

(b) $H^{\prime \prime}$ has order 2 and $|Z| \equiv 2 \bmod 4$ and $H / Z \cong A_{4}$, or

(c) $H^{\prime \prime} \cong Q_{8}$ and $|Z| \equiv 2 \bmod 4$ and $H / Z \cong S_{4}$, or

(d) $\quad H^{\prime \prime} \cong \operatorname{SL}(2,5)$ and $\left|H: H^{\prime \prime} Z\right| \leq 2$;

see $[12,6.1 .9,6.1 .11],[7,18.2]$ for the solvable case, and $[12,6.3 .1]$ or $[7,18.6]$ for the nonsolvable case (or [13, Sätze 6, 8, 16]).

All subgroups of order 3 are conjugate in $G$, by 2-finiteness and Sylow's theorem. According to Zhurtov [16, Lemma 8], $G$ contains at most one subgroup isomorphic to $\mathrm{SL}(2,5)$; this follows also from [15, Theorem 1] or [9, Theorem 1]. Hence the subgroup $T$ generated by all copies of $\operatorname{SL}(2,5)$ and all involutions in $G$ is finite and normal in $G$. Below we show that the quotient $\Gamma:=G / T$ is locally finite (which implies that $G$ is locally finite).

Every finite subgroup $\widetilde{H}$ of $\Gamma$ has a normal subgroup $\widetilde{Z}$ such that all Sylow subgroups of $\widetilde{Z}$ are cyclic and $\widetilde{H} / \widetilde{Z}$ is isomorphic to a quotient of $A_{4}$ or $S_{4}$, hence a $\{2,3\}$-group. Repeatedly applying [8, 10.1.9] we obtain for $n \geq 5$ that the set $\widetilde{H}_{n}=\{h \in \widetilde{H} \mid$ no prime divisor of $|\langle h\rangle|$ is smaller than $n\} \subseteq \widetilde{Z}$ is a subgroup of $\widetilde{H}$. By 2-finiteness, this property of $\widetilde{H}$ carries over to $\Gamma$, and the set

$$
\Gamma_{n}=\{g \in \Gamma \mid \text { no prime divisor of }|\langle g\rangle| \text { is smaller than } n\}
$$

is a (normal) subgroup of $\Gamma$ for every integer $n \geq 5$.

We claim that $\mathrm{O}(\Gamma)$, the largest normal subgroup of $\Gamma$ consisting of elements of odd order, is locally finite. For every prime $p$, all $p$-sections of $\mathrm{O}(\Gamma)$ are locally cyclic (by 2-finiteness). We have $\Gamma_{5} \subseteq \mathrm{O}(\Gamma)$, and $\mathrm{O}(\Gamma) / \Gamma_{5}$ is a locally cyclic 3 -group. For $n \geq 5$ the quotient $\Gamma_{n} / \Gamma_{n+1}$ is a $p$-group (in fact, 
trivial unless $n=p$ is a prime) which is locally cyclic, as well. Since local finiteness is an extension property (see $[8,14.3 .1]$ ), the quotient $\mathrm{O}(\Gamma) / \Gamma_{n}$ is locally finite for $n \geq 5$. All Sylow subgroups of a finite subgroup $S$ of $\mathrm{O}(\Gamma) / \Gamma_{n}$ are cyclic, hence $S^{\prime \prime}=\{1\}$ by a result of Hölder, Burnside and Zassenhaus, see $[8,10.1 .10]$ or $[12,5.4 .1]$ or $[13$, Satz 5$]$. Since $\mathrm{O}(\Gamma) / \Gamma_{n}$ is locally finite, we infer that $\mathrm{O}(\Gamma)^{\prime \prime} \subseteq \Gamma_{n}$ for $n \geq 5$, hence $\mathrm{O}(\Gamma)^{\prime \prime}$ is trivial. This implies that $\mathrm{O}(\Gamma)$ is locally finite (because periodic abelian groups are locally finite and local finiteness is an extension property).

Now we claim that the $\{2,3\}$-group $\bar{\Gamma}:=\Gamma / \mathrm{O}(\Gamma)$ is locally finite. Suppose $x \in \bar{\Gamma}$ has order $3 k$ with $k \in\{2,3\}$. By definition of $\mathrm{O}(\Gamma)$, the subgroup $\left\langle x^{k}\right\rangle$ of order 3 is not normal in $\bar{\Gamma}$, hence $\left\langle x^{k}\right\rangle \neq\left\langle y^{k}\right\rangle$ for some conjugate $y$ of $x$. The finite group $\bar{H}=\langle x, y\rangle$ has type (b) or (c), since subgroups of $\bar{\Gamma}$ of type (a) have a normal Sylow 3 -subgroup by $[8,10.1 .9]$ and therefore only one subgroup of order 3. The corresponding subgroup $\bar{Z}$ has odd order, hence $\bar{Z}=\mathrm{O}(\bar{H})$ is a 3-group. For $k=3$ we obtain $\left\langle x^{3}\right\rangle=\bar{Z}=\left\langle y^{3}\right\rangle$, which is a contradiction. For $k=2$ we have $x^{3} \notin \bar{Z}$, hence $x^{2} \in \bar{Z}$, and analogously $y^{2} \in \bar{Z}$; hence $\bar{H} / \bar{Z} \cong A_{4}, S_{4}$ is generated by two involutions, which is absurd. Thus $\bar{\Gamma}$ has no element of order 6 or 9 , and $\bar{Z}=\mathrm{O}(\bar{H})$ is trivial for finite subgroups $\bar{H}$ of $\bar{\Gamma}$ of type (b), (c).

By Zhurtov [16, Lemma 8], the elements of order 3 in $\bar{\Gamma}$ generate a locally finite (normal) subgroup; since the quotient is a 2-group which is locally finite (any two squares commute), this implies that $\bar{\Gamma}$ is locally finite. We offer the following alternative argument. If $x, y \in \bar{\Gamma}$ are 2-elements and $\langle x, y\rangle$ is of type (a), then $x^{2}$ and $y^{2}$ centralize the 3-group $\mathrm{O}(\langle x, y\rangle)$ and belong to a cyclic group of squares of 2-elements. Thus the set $\Delta:=\left\{x^{2} \mid x \in \bar{\Gamma}\right.$ is a 2-element $\}$ is an abelian (normal, locally finite) subgroup of $\bar{\Gamma}$. Every element of $\bar{\Gamma} / \Delta$ has order 1,2 or 3 , hence $\bar{\Gamma} / \Delta$ is locally finite by a theorem of B. H. Neumann [5] (in fact, $\bar{\Gamma} / \Delta$ is finite by [4, Lemma 2.4], since its 2 -subgroups have order at most 4). Thus $\bar{\Gamma}$ is locally finite.

We conclude that $\Gamma=G / T$ is locally finite, and so is $G$, as $T$ is finite. Every finite subgroup $H$ of the locally finite group $G$ satisfies $\left|H^{\prime \prime}\right| \leq 120$, hence $G^{\prime \prime}$ is finite and coincides with one of the groups $H^{\prime \prime}$ listed above.

The finite subgroups of the abelian groups $G / G^{\prime}$ and $G^{\prime} / G^{\prime \prime}$ are cyclic or have a cyclic subgroup of index 2 . Hence $G / G^{\prime}$ and $G^{\prime} / G^{\prime \prime}$ have locally cyclic subgroups of index at most 2. This implies that $G / G^{\prime}$ and $G^{\prime} / G^{\prime \prime}$ are countable, and so is $G$, as $G^{\prime \prime}$ is finite.

Proof of Corollary 2. The multiplicative group $N^{\times}$acts faithfully on the additive group $(N,+)$ as a fixed-point-free automorphism group. By Theorem 1, $N^{\times}$is locally finite. This implies that $N$ is a locally finite nearfield; see Wähling [10, Satz 2].

Proof of Corollary 3. According to the theorem in Collins [2], $G$ contains a sharply transitive abelian normal subgroup $(N,+)$. Thus $G$ is a semidirect product $N G_{0}$ where $G_{0} \leq \operatorname{Aut}(N,+)$ is fixed-point-free (and transitive on the 
set $N \backslash\{0\})$. Therefore $G=\{x \mapsto a \circ x+b \mid a, b \in N, a \neq 0\}$ for a suitable nearfield-multiplication $\circ$ on $(N,+)$. Since $G_{0} \cong(N \backslash\{0\}, \circ)$ is 2-finite, Corollary 2 implies that the nearfield $(N,+, \circ)$ is locally finite.

Every infinite, locally finite nearfield is a 'regular' nearfield constructed from a locally finite field; see [3, Theorem 2.2] and its proof, or [11, IV, 9.5a]. This means that there exists a field multiplication $\cdot$ such that $F=(N,+, \cdot)$ is a locally finite field and for $a, x \in N$ one has $a \circ x=a \cdot \alpha(x)$, where $\alpha \in \operatorname{Aut} F$ depends on $a$ only.

Monstrous examples. Let $p>2$ be a prime number. For every integer $t$ such that $p^{t}$ is sufficiently large, there exists a finitely generated infinite group $G$ of exponent $p^{t}$ such that the center $C$ of $G$ has order $p$ and contains all elements of order $p$ in $G$; see [6, Theorems 31.2, 31.3, 31.5 and (the proof of) 31.7]. If $t=2$ and if the prime $p$ is sufficiently large, the quotient $G / C$ is a so-called Tarski monster: it is infinite, simple and all its proper nontrivial subgroups are cyclic of order $p$ (see [6, Section 28]).

Let $C=\langle c\rangle$ and let $I$ be the ideal generated by the central element $1+$ $c+\cdots+c^{p-1}$ in the rational group ring $\mathbb{Q} G$. The natural action of $G$ on $\mathbb{Q} G$ yields a faithful action of $G$ on $\mathbb{Q} G / I$ which is fixed-point-free, because every element $a+I \in \mathbb{Q} G / I$ fixed by a nontrivial element of $G$ is fixed also by $c$, hence $p a \in a+c a+\cdots+c^{p-1} a+I \subseteq I a+I=I$ and $a \in I$.

This action of $G$ is not transitive on the non-zero elements of $\mathbb{Q} G / I$; indeed, a non-zero element cannot be mapped to its negative (as $G$ contains no involution), and $1+I$ cannot be mapped to $1-c+I$.

\section{References}

[1] P. M. Cohn, Skew fields, Cambridge University Press 1995.

[2] M. J. Collins, Some infinite Frobenius groups, J. Algebra 131 (1990), 161-165.

[3] S. Dancs Groves, Locally finite nearfields, Abh. Math. Sem. Univ. Hamburg 48 (1977), 89-107.

[4] E. Jabara And P. Mayr, Frobenius complements of exponent dividing $2^{m} \cdot 9$, Forum Math. 21 (2009), 217-220.

[5] B. H. Neumann, Groups whose elements have bounded orders, J. London Math. Soc. 12 (1937), 195-198.

[6] A. Yu. Ol'shanskiI, Geometry of defining relations in groups, Translated from the 1989 Russian original. Kluwer, Dordrecht 1991.

[7] D. Passman, Permutation groups, Benjamin, New York 1968.

[8] D. J. S. Robinson, A course in the theory of groups, Springer-Verlag, Berlin 1982.

[9] A. I. Sozutov, On the structure of the noninvariant factor in some Frobenius groups, Siberian Math. J. 35 (1994), 893-901.

[10] H. WäHLING, Lokal endliche, scharf zweifach transitive Permutationsgruppen, Abh. Math. Sem. Univ. Hamburg 56 (1986), 107-113.

[11] H. WäHLing, Theorie der Fastkörper, Thales Verlag, Essen 1987. 
[12] J. A. Wolf, Spaces of constant curvature, McGraw-Hill, New York 1967.

[13] H. Zassenhaus, Über endliche Fastkörper, Abh. Math. Sem. Univ. Hamburg 11 (1935), 187-220.

[14] A. Kh. Zhurtov, Quadratic automorphisms of abelian groups, Algebra and Logic 39 (2000), 184-188.

[15] A. Kh. Zhurtov, On regular automorphisms of order 3 and Frobenius pairs, Siberian Math. J. 41 (2000), 268-275.

[16] A. Kн. Zhurtov, On a group acting locally freely on an abelian group, Siberian Math. J. 44 (2003), 275-277.

\section{Theo Grundhöfer}

Institut für Mathematik,

Universität Würzburg,

Am Hubland,

97074 Würzburg, Germany

e-mail: grundh@mathematik.uni-wuerzburg.de

ENRICO JABARA

Dipartimento di Filosofia e Beni Culturali,

Università di Ca' Foscari,

Dorsoduro 3484/d,

30123 Venetia, Italy

e-mail: jabara@unive.it

Received: 28 March 2011 